\title{
Ferromagnetism mediated by few electrons in a semimagnetic quantum dot.
}

\author{
J. Fernández-Rossier ${ }^{1}$, L. Brey ${ }^{2}$ \\ (1)Departamento de Física Aplicada, Universidad de Alicante, 03690 San Vicente del Raspeig, Spain \\ (2) Insituto de Ciencia de Materiales de Madrid, CSIC, Cantoblanco, 28049 Madrid, Spain
}

(Dated: November 4, 2018)

\begin{abstract}
A (II,Mn)VI diluted magnetic semiconductor quantum dot with an integer number of electrons controlled with a gate voltage is considered. We show that a single conduction band electron is able to induce a spontaneous collective magnetization of the Mn spins, overcoming the short range antiferromagnetic interactions. The carrier mediated ferromagnetism in the dot survives at temperatures above 1 Kelvin, two orders of magnitude larger than the Curie temperature for the same material in bulk. The magnetic behavior of the dot depends dramatically on the parity of the number of injected electrons.
\end{abstract}

PACS numbers:

Introduction. The range, strength and sign of exchange interactions between magnetic impurities in diluted magnetic semiconductors (DMS) depends on the density and nature of the states at the Fermi level. Mn doped semiconductors of the families (II,Mn)-VI and (III,Mn)-V order ferromagnetically in the presence of carriers that mediate indirect exchange interactions between Mn. In the case of (III,Mn)V compounds like GaAsMn, Mn acts as an acceptor supplying holes responsible of the ferromagnetism below a transition temperature which depends on both $\mathrm{Mn}$ and hole densities $\underline{\underline{\underline{1}}}$ and can reach 160 $\mathrm{K}^{2}$. In contrast, $\mathrm{Mn}$ does not supply itinerant carriers in (II,Mn)VI compounds. These materials do not order ferromagnetically ${ }^{3}$ unless further doping with acceptors provides holes which produce ferromagnetism below a carrier density dependent Curie Temperature $\left(T_{C}\right)$ of approximately $2 \mathrm{~K}^{4}$.

Electrical control of the carrier density, in contrast with chemical doping, has been demonstrated in a number of DMS heterostructures, making it possible to alter reversibly properties of the material like the $T_{C}{ }^{5.6}$ and the coercive field ${ }^{7}$ of these systems. The fact that the carrier density is much higher than the relative change achieved artificially sets limits to the control. In contrast, the number of electrons in a GaAs quantum dot can be varied one by one, starting from zero, in single electron transistors ${ }^{8}$. Single electron transistors with non magnetic II-VI (CdSe) quantum dots of lateral size smaller than $10 \mathrm{~nm}$ have been also fabricated ${ }^{9}$. (II,Mn)VI quantum dots of similar size have also been grown and studied magneto-optically by several groups $\frac{10}{}$. Therefore, the fabrication of a single electron transistor with (II,Mn)VI quantum dots seems feasible and motivates this work.

We study the magnetic properties of a (II-VI)Mn quantum dot with an integer number of electrons. The lateral dimensions of the dot are smaller than $10 \mathrm{~nm}$ and, for the range of Mn concentration considered, $x<0.05$, the number of $\mathrm{Mn}$ atoms in the dot is of the order of one hundred and the number of electrically injected electrons ranges from zero to 10 . We find compelling theoretical and numerical evidence that the addition of a single electron to an otherwise paramagnetic DMS dot is enough to couple most of the Mn spins so that a net total magnetic moment emerges spontaneously. Therefore, a single electron transistor with a DMS quantum dot would switch on and off the magnetic total magnetic completely in a controlled and reversible manner.

Hamiltonian. The Hamiltonian describing the system is the zero dimensional version of the standard exchange Hamiltonian used for both bulk ${ }^{3.11}$ and two dimensional systems 12 . For simplicity we only consider conduction band electrons, for which we can ignore spin orbit interaction, and we ignore Coulomb repulsion. The latter tends to enhance carrier mediated exchange interactions so that our results do not change qualitatively if this approximation is abandoned. Conduction band electrons (creation operator $c_{n, \sigma}^{\dagger}$ ) occupy confined levels $\epsilon_{n}^{0}$ of the dot. The Mn spins are described with $S=5 / 2$ operators $\vec{M}_{I}$. The spin of the quantum dot electrons and the $\mathrm{Mn}$ interact via a zero range exchange interaction. The Mn spins interact also with each other via a short range antiferromagnetic superexchange interaction ${ }^{3} \mathcal{J}_{I, I^{\prime}}$. The Hamiltonian reads:

$$
\mathcal{H}=\sum_{n, \sigma} \epsilon_{n}^{0} c_{n, \sigma}^{\dagger} c_{n, \sigma}-J_{c} \sum_{I} \vec{M}_{I} \cdot \vec{S}_{e}\left(\vec{x}_{I}\right)+\mathcal{H}_{A F}
$$

where $\mathcal{H}_{A F}=\frac{1}{2} \sum_{I, I^{\prime}} \mathcal{J}_{I I^{\prime}} \vec{M}_{I} \cdot \vec{M}_{I^{\prime}}$ and

$$
\vec{S}\left(\vec{x}_{I}\right) \equiv \sum_{\sigma, \sigma^{\prime}, n, n^{\prime}} \phi_{n}^{*}\left(\vec{x}_{I}\right) \phi_{n^{\prime}}\left(\vec{x}_{I}\right) \frac{1}{2} \vec{\tau}_{\sigma, \sigma^{\prime}} c_{n, \sigma}^{\dagger} c_{n^{\prime}, \sigma^{\prime}}
$$

is the quantum dot electron local spin density and $\phi_{n}\left(\vec{x}_{I}\right)$ is the $n^{\text {th }}$ orbital wave functions of dot. Exchange interaction produces transitions between different levels of the dot. The interlevel spacings considered here are of the order of $20 \mathrm{meV}$ and higher, except for the degeneracies that some of the dots might have. The exchange energy of a quantum dot electron with a single Mn is approximately given by $j \equiv J_{c} / \Omega_{D}$, where $\Omega_{D}$ is the quantum dot volume. For $\mathrm{Cd}_{1-x} \mathrm{Mn}_{x}$ Te quantum dots with $10 \mathrm{~nm}$ of lateral dimension, we have $j \simeq 1.5 \times 10^{-2} \mathrm{meV}$, much smaller than the level spacing.

Effective spin Hamiltonian. In the following we derive analitycally the effective interaction between the $\mathrm{Mn}$ 
spins, which are treated classically. The canonical ensemble equilibrium partition function for the dot with a fixed number of electrons $N_{e}$ is given by:

$$
\mathcal{Z}_{N}=\int d \vec{M}_{1} . . d \vec{M}_{N} e^{-\beta \mathcal{H}_{A F}} \sum_{\alpha} e^{-\beta E_{\alpha, N}\left(\vec{M}_{I}\right)}
$$

where $\beta=1 / k_{B} T$ and $E_{\alpha, N_{e}}\left(\vec{M}_{I}\right)$ is the energy of the Slater determinant labeled with $\alpha$, for a given Mn spin configuration, $\left(\vec{M}_{I}\right)$. At low temperatures we can drop from the sum over configurations in (3) all except the ground state Slater $\alpha=0$, whose energy we denote with $\mathcal{E} \equiv E_{0, N_{e}}\left(\vec{M}_{I}\right)$. In this approximation the effective Mn-Mn coupling is:

$$
\mathcal{H}_{\text {eff }}=\mathcal{H}_{A F}+\mathcal{E}\left(\vec{M}_{I}\right)
$$

Let $\lambda_{l}\left(\vec{M}_{I}\right)$ be the eigenstates of the Hamiltonian matrix

$$
\mathcal{H}_{n \sigma, n^{\prime} \sigma^{\prime}} \equiv \epsilon_{n}^{0}-\frac{J_{c}}{2} \sum_{I} \vec{M}_{I} \cdot \vec{\tau}_{\sigma, \sigma^{\prime}} \phi_{n}^{*}\left(\vec{x}_{I}\right) \phi_{n^{\prime}}\left(\vec{x}_{I}\right)
$$

associated to (11) for a given configuration of classical spins, $\vec{M}_{I}$. This matrix has $n$ intra-level $2 \times 2$ boxes. For the small dots considered here, the leading order term comes from the intra-level term. To linear order in $J_{c}$ we have: $\lambda_{l} \simeq \epsilon_{n}^{0} \pm\left.\left|\frac{J_{c}}{2} \sum_{I}\right| \phi_{n}\left(\vec{x}_{I}\right)\right|^{2} \vec{M}_{I} \mid$. The many-electron ground state energy is the sum over the $N_{e}$ lowest eigenvalues $\lambda_{l}$. For an odd number of electrons, $N_{e}=2 N+1$, all the contributions linear in $J_{c}$ coming from the first $2 N$ electrons vanish identically and the only contribution comes from the most external electron. Modulo an irrelevant constant, we obtain the following ground state electronic energy for a dot with $2 N+1$ electrons:

$$
\mathcal{E}_{o}=-\sqrt{\frac{J_{c}^{2}}{4} \sum_{I, I^{\prime}}\left|\phi_{N+1}\left(\vec{x}_{I}\right)\right|^{2}\left|\phi_{N+1}\left(\vec{x}_{I^{\prime}}\right)\right|^{2} \vec{M}_{I} \cdot \vec{M}_{I^{\prime}}}
$$

Equations (4) and (6) define the carrier mediated MnMn coupling which is one of the important results of the paper. The Mn-Mn interaction mediated by an odd number of electrons, including a single electron, always favors ferromagnetic couplings and it scales with $J_{c}$. These features are in contrast with the standard bulk RKKY coupling, which scales with $J_{c}^{2}$, and it can be either positive or negative. For these reasons the effective coupling (6) is stronger than its bulk counterpart. There is yet another difference: RKKY coupling involves a sum over several orbital states whereas (6) only depends on the most external orbital state, opening the door to a quantum design of indirect exchange interactions in confined geometries 14

For an even number of electrons all the contributions linear in $J_{c}$ cancel. The leading order contribution to $\mathcal{E}_{\rceil}$comes from inter-level exchange coupling. We calculate $\mathcal{E}_{\rceil}$doing perturbation theory around $J_{c}=0$. The magnetic part of the ground state electronic so obtained reads:

$$
\mathcal{E}_{e}=\sum_{I, I^{\prime}}\left[\frac{J_{c}^{2}}{2} \sum_{n, n^{\prime}} \gamma_{n, n^{\prime}}\left(I, I^{\prime}\right) \frac{f_{n}-f_{n^{\prime}}}{\epsilon_{n}^{0}-\epsilon_{n^{\prime}}^{0}}\right] \vec{M}_{I} \cdot \vec{M}_{I^{\prime}}
$$

where $\gamma_{n, n^{\prime}}\left(I, I^{\prime}\right) \equiv \phi_{n}^{*}\left(\vec{x}_{I}\right) \phi_{n^{\prime}}\left(\vec{x}_{I}\right) \phi_{n}^{*}\left(\vec{x}_{I^{\prime}}\right) \phi_{n^{\prime}}\left(\vec{x}_{I}^{\prime}\right)$ and $f_{n}=0,1$ are the occupation of the unperturbed dot in the ground state electronic configuration. The effective couplings (7) are weaker than (6) and can be both positive and negative (ferro or antiferromagnetic) for a given dot and different Mn couples. The striking differences between effective interactions (6) and (7) permit to anticipate very different behavior for dots with open and closed shell electronic structure.

Local mean field theory. Effective interactions (6) and (7) between the spins result from integrating out the conduction electrons in some limits and treating the $\mathrm{Mn}$ spins classically. We now do a mean field theory for Hamiltonian (11), keeping track of both $\mathrm{Mn}$ and electrons. Quantum dot electrons interact with an effective exchange field provided by the expectation value of the Mn spin operators. The latter is calculated assuming that each Mn spin interact with an effective field provided by the quantum dot spin density and its neighboring Mn spins, via antiferromagnetic superexchange interaction. Since both the electron spin density and the Mn neighborhood are different for each $\mathrm{Mn}$, each Mn has a different expectation value, in contrast with the homogeneous models used for bulk ${ }^{3,11}$. The local mean field felt by the Mn at $\vec{x}_{I}$ reads:

$$
g \mu_{B}\left\langle\overrightarrow{\mathcal{B}}_{I}\right\rangle=J_{c}\left\langle\vec{S}\left(\vec{x}_{I}\right)\right\rangle-\frac{1}{2} \sum_{I^{\prime}} \mathcal{J}_{I I^{\prime}}\left\langle\vec{M}_{I^{\prime}}\right\rangle
$$

The expectation value for the magnetization of a spin $S=5 / 2$ in the effective field of eq. (8) reads:

$$
\left\langle\vec{M}_{I}\right\rangle=S \vec{u}_{I} B_{S}\left(\frac{S}{k_{B} T} g \mu_{B}\left\langle\overrightarrow{\mathcal{B}}_{I}\right\rangle\right)
$$

where $\vec{u}_{I}$ the unitary vector parallel to $\left\langle\overrightarrow{\mathcal{B}}_{I}\right\rangle$ and $B_{S}$ is the Brillouin function ${ }^{3}$. Finally, the equation for the average conduction electron local spin density is:

$$
\left\langle\vec{S}\left(\vec{x}_{I}\right)\right\rangle=\frac{1}{Z_{e l}} \sum_{\alpha} e^{-\beta E_{\alpha}} \sum_{l}\left\langle\Phi_{l}\left|\vec{S}\left(\vec{x}_{I}\right)\right| \Phi_{l}\right\rangle f_{l}(\alpha)
$$

where $Z_{e l}=\sum_{\alpha} e^{-\beta E_{\alpha}}$ and $\left|\Phi_{l}\right\rangle$ is eigenvector associated to the $\lambda_{l}$ eigenvalue of Hamiltonian $\left[5\right.$ with $\vec{M}_{I}$ replaced by $\left\langle\vec{M}_{I}\right\rangle$ given by eq. (9) and $f_{l}(\alpha)$ is the occupation $(0$ or 1) level in the many electron configuration $\alpha$.

In the following we show results for $\mathrm{Cd}_{1-x} \mathrm{Mn}_{x} \mathrm{Te}$ hard wall cubic quantum dots of dimensions $L_{x}, L_{y}$ and $L_{z}$ for which $\epsilon_{n}^{0}$ and $\phi_{n}$ are known analytically. Following reference 3 we take $J_{c}=15 \mathrm{eV} \AA^{3}$. Superexchange antiferromagnetic coupling $\mathcal{J}_{I I^{\prime}}$ decays exponentially with distance and we only consider coupling to first neighbours, 
$\mathcal{J}_{F N}=0.5 \mathrm{meV}^{3,13}$. The positions of the Mn atoms are randomly chosen in the cation sites of the Zinc Blende lattice with lattice constant, $a=6.4 \AA$, avoiding double occupation of the sites. The initial value of $\left\langle\vec{M}_{I}\right\rangle$ is also chosen randomly. Self consistent numerical solution of equations (8), (9) and (10) yield converged values of $\left\langle\vec{M}_{I}\right\rangle$ which are stable, independent of all initial conditions, convergence procedure and small variations of the energy scales of the problem. We characterize the collective magnetic order with the average Mn magnetization per Mn:

$$
\langle M\rangle \equiv \sqrt{\left|\frac{1}{N} \sum_{I}\left\langle\vec{M}_{I}\right\rangle\right|^{2}}
$$

which can range between 0 and $S=5 / 2$. Different realizations of the Mn positional configurations, $\left\{\vec{r}_{I}\right\}$ for otherwise identical dots give different values of $\langle M\rangle$. Experiments with a single dot are feasible in principle, so that $\langle M\rangle$ is observable for a single dot. To make sure that general results are independent of a particular $\mathrm{Mn}$ realization we perform averages over different realizations of $\left\{\vec{r}_{I}\right\}$. The net magnetization of a dot averaged over configurations is denoted by $\langle\langle M\rangle\rangle$. The mean standard deviation among different realizations of $\left\{\vec{r}_{I}\right\}$ is denoted by $\sigma_{M}$.
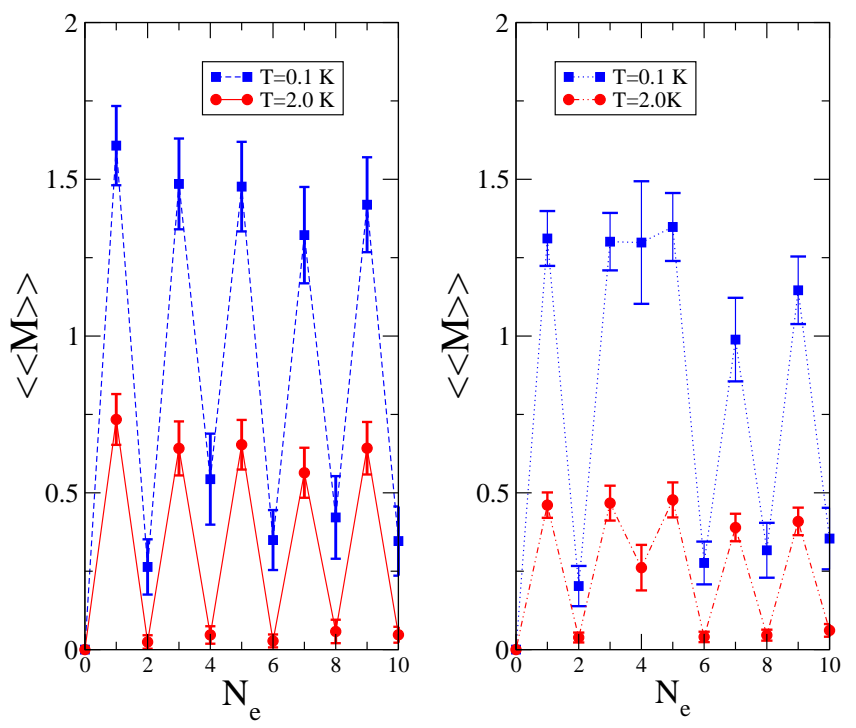

FIG. 1: (Color online). Magnetization per Mn versus $N_{e}$, averaged over disorder configuration for two different dots.

In figure 1 we plot $\langle\langle M\rangle\rangle$ and $\sigma_{M}$ (vertical bars) as a function of $N_{e}$ for two different dots at two different temperatures, $k_{B} T=0.1 \mathrm{~K}$ and $2.0 \mathrm{~K}$. Dots 1 and 2 (left and right panels) have the same $\mathrm{Mn}$ concentration and the same $L_{x}\left(x=0.01, L_{x}=4 \mathrm{~nm}\right)$ but different dimensions. Dot 1 has $L_{y}=6 \mathrm{~nm}, L_{z}=7 \mathrm{~nm}$ and dot 2 has $L_{y}=8.5$ $\mathrm{nm}, L_{z}=9 \mathrm{~nm}$. The number of Mn impurities is 25 and 46 respectively. We find that: i) A single electron is enough to induce a spontaneous collective magnetization different from zero in the absence of applied field. The collective magnetization survives at temperatures of 1 Kelvin and higher. The bulk mean field Curie Temperature for parabolic bands yields, for $\mathrm{Cd}_{0.99} \mathrm{Mn}_{0.01} \mathrm{Te},\left.k_{B} T_{C}\right|_{\text {Bulk }}=\frac{S(S+1)}{3} \frac{3}{2} c_{\mathrm{Mn}} \frac{n}{\epsilon_{F}} \simeq 20$ $\mathrm{mK}$ where $n$ and $c_{\mathrm{Mn}}$ are the electron and Mn density respectively corresponding to the dot of figure 1 . Therefore, carrier mediated spin correlations in the quantum dots survive at temperatures 2 orders or magnitude larger than $T_{C}$ in bulk.

ii) The addition or removal of a single electron produces a dramatic change in the magnetization of the dots.

iii) The spontaneous magnetization is larger, in general, for open shell configurations (odd number of electrons) than for closed shell configurations (even number of electrons). Closed shell configurations with a small gap can yield larger inter-level couplings. This is the case of $N_{e}=4$, specially in dot 2 with smaller energy level spacings than dot 1.
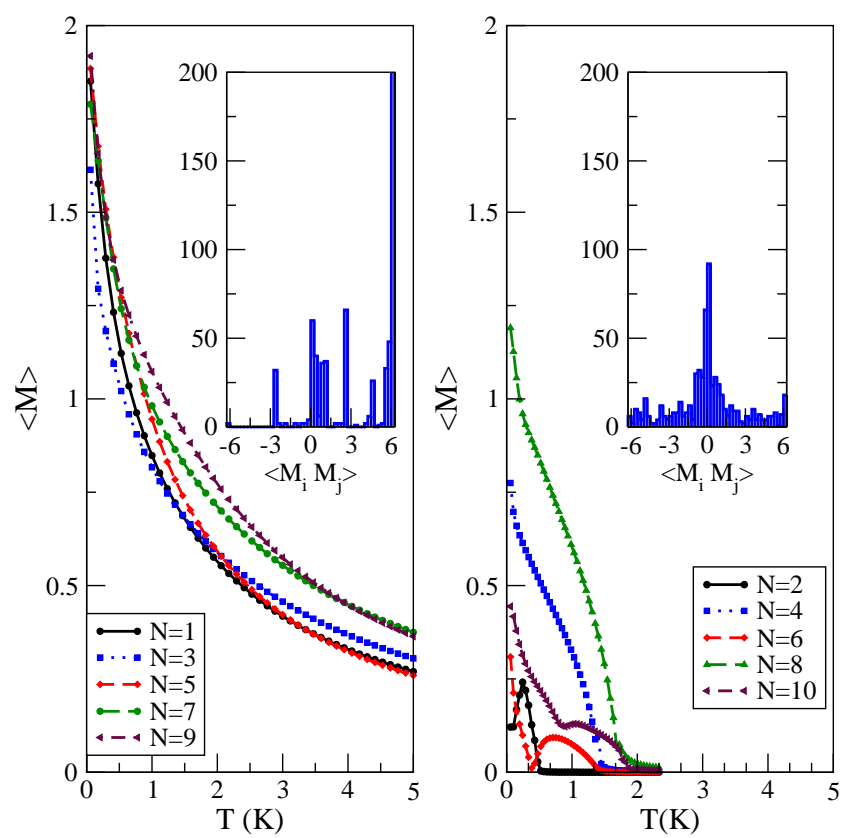

FIG. 2: (Color online). Magnetization per Mn vs temperature for odd (left) and even (right) number of electrons for dot 1 (see text). In the inset we show the histograms of $\left\langle\vec{M}_{i} \cdot \vec{M}_{j}\right\rangle$ at $k_{b} T=50 \mathrm{mK}$ for $\mathrm{N}=1$ (left panel) and $\mathrm{N}=2$ right panel

In figure 2 we show $M(T)$ for a single Mn realization for dot 1 . In the left (right) panel of fig. 2 we show $M(T)$ for an odd (even) number of electrons. The shape of the curves for $N_{e}$ odd are all very similar to each other and resemble the spontaneous magnetization of a confined exciton-polaron ${ }^{15}$. In contrast, the shape for the $M(T)$ curves varies from case to case for even $N_{e}$ and results from the complicated competition of carrier mediated ferro and antiferromagnetic couplings and superexchange. To quantify this, we plot in the insets his- 


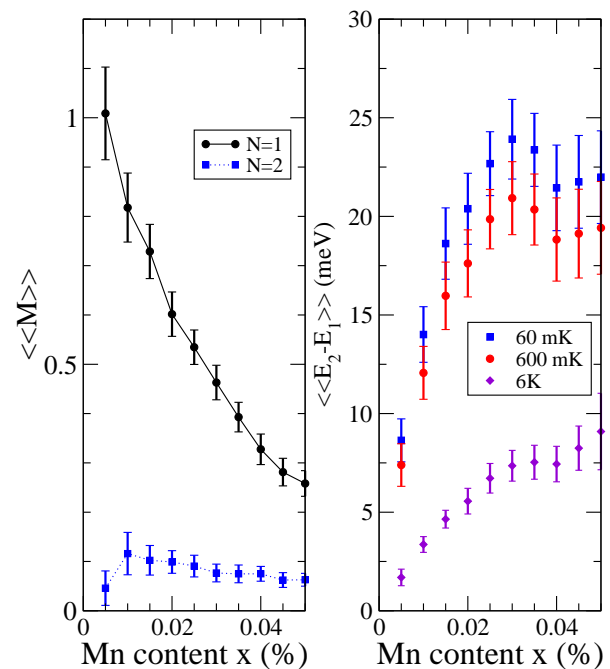

FIG. 3: (Color online). Left panel: $\langle\langle M\rangle\rangle$ per Mn as a function of Mn content, $x$ at $k_{B} T=0.6$ Kelvin. Right panel: spontaneous spin splitting of the lowest level of the dot, at three temperatures, as a function of Mn content $x$.

tograms of the values of the correlation matrix $\left\langle\vec{M}_{I} \cdot \vec{M}_{J}\right\rangle$ for $N_{e}=1$ (left) and $N_{e}=2$ (right) at very low temperatures $(50 \mathrm{mK})$. The $N_{e}=1$ histogram is biased towards positive values (ferromagnetic couplings) whereas the $N_{e}=2$ histogram displays a rather symmetric distribution of ferromagnetic and antiferromagnetic couplings.

The competition between carrier mediated and superexchange couplings evolves as the Mn concentration increases. In the left panel of figure 3 we show $\langle\langle M\rangle\rangle$ and $\sigma_{M}$ (vertical bars) as a function of Mn concentration, $x$, for dot 2. We see that, for $N_{e}=1,\langle\langle M\rangle\rangle$ decreases monotonically as a function of $x$ as a result of the increase of the number first neighbour pairs coupled antiferromagnetically. The curve for $N_{e}=2$ has a maximum around $x=0.01$ and decreases at higher concentrations.

The spin rotational symmetry of Hamiltonian (1) makes any direction in the spin space equivalent. Therefore, when a spontaneous magnetic moment arises from carrier mediated $\mathrm{Mn}-\mathrm{Mn}$ interactions, it can point in any direction of the space. The effects related to spontaneous magnetization can be observed provided that the collective magnetization is static during the probe time scale. A small source of spin anisotropy, like an applied field or Rashba spin orbit interaction, can be very efficient in slowing down the magnetization dynamics. The spontaneous magnetization results in a splitting of the quantum dot energy levels which could be measured in transport ${ }^{8}$. In the right panel of fig. 3 we plot the energy splitting of the lowest quantum dot doublet as a function of $x$, for $N_{e}=1$ at three different temperatures for dot 2. The splitting is a decreasing function of temperature and an increasing function of the $\mathrm{Mn}$ content up to $x=0.03$, declining for higher $x$, due to the increase of first neighbours pairs.

In summary, our results indicate that a very large control of carrier mediated interactions can be achieved in quantum dots in which the number of electrons can be changed one by one. We claim that a few conduction band electrons couple the spin of several tens of $\mathrm{Mn}$ atoms in a (II,Mn) VI semiconductor quantum dot. An odd number of electrons, including just one, yield a ferromagnetic coupling (eq. 6) and left panels of figures 1 and 2 ) whereas and even number of electrons give both ferro and antiferromagnetic carrier mediated couplings (eq. 7 and right panels of figures 1 and 2). The result of the competition between carrier mediated interactions and short range antiferromagnetic superexchange is a spontaneous collective magnetization which survives at temperatures of the order of 1 Kelvin, 2 orders of magnitude higher than the mean field prediction for the same material in bulk. The resulting spontaneous spin splitting of the energy levels of the dot could be measured in transport experiments.

We acknowledge fruitful discussions with J.J. Palacios and E. Louis. Financial support is acknowledged from Grants No MAT2002-04429-c03-01, MAT2003-08109C02-01, Ramon y Cajal Program (MCyT,Spain), Fundación Ramón Areces, and UA/GRE03-15. This work has been partly funded by FEDER funds.
1 H. Ohno, Science 281, 951 (1998).

2 K. W. Edmonds et al., Phys. Rev. Lett. 92, 037201 (2004). D. Chiba et al., Appl. Phys. Lett. 82, 3020 (2003);

3 J. K. Furdyna, J. Appl. Phys 64 R29 (1988).

4 A. Haury et al., Phys. Rev. Lett. 79, 511 (1997) D. Ferrand et al.,Phys. Rev. B 63, 085201 (2001)

${ }^{5}$ H. Ohno et al., Nature, 408, 944 (2000)

${ }^{6}$ H. Boukari et al., Phys. Rev. Lett. 88, 207204 (2002).

7 D.Chiba et al., Science 301, 943 (2003)

8 L.P. Kouwenhoven, D.G. Austing, Reports on Progress in Physics 64701 (2001)

9 D. Klein et al., Nature 389, 699 (1997)

10 A. A. Maksimov et al. Phys. Rev. B62, R7767 (2000) G. Bacher et al. Phys. Rev. Lett. 89, 127201 (2002) G. Bacher et al. Appl. Phys. Lett. 79, 524 (2001)
11 T. Dietl et al., Science 2871019 (2000).

12 B. Lee, T. Jungwirth, and A. H. MacDonald Phys. Rev. B 61, 15606 (2000) L. Brey and F. Guinea Phys. Rev. Lett. 85, 2384 (2000) J. Fernández-Rossier and L. J. Sham Phys. Rev. B 64, 235323 (2001) J. Fernández-Rossier and L. J. Sham Phys. Rev. B 66, 073312 (2002)

13 Y. Shapira, Jr. and N. F. Olivera, Phys. Rev. B 35, 6888 (1987).

14 H.C. Manoharan, C.P. Lutz, and D.M. Eigler, Nature 403, 512 (2000). D. Porras, J. Fernández-Rossier, and C. Tejedor Phys. Rev. B 63, 155406 (2001)

15 A. K. Bhattacharjee and C. Benoit a la Guillaume. Phys. Rev. B55,10613 (1997) 